\title{
The Magnetofection Method: Using Magnetic Force to Enhance Gene Delivery
}

\author{
Christian Plank ${ }^{1, *}$, Ulrike Schillinger ${ }^{1}$, Franz \\ Scherer ${ }^{1}$, Christian Bergemann², Jean-Serge \\ Rémy ${ }^{3}$, Florian Krötz ${ }^{4}$, Martina Anton', Jim \\ Lausier $^{5}$ and Joseph Rosenecker ${ }^{5}$ \\ ${ }^{1}$ Institute of Experimental Oncology, Technical \\ University Munich, Ismaninger Str. 22, D-81675 Munich, \\ Germany \\ ${ }^{2}$ chemicell, D-10823 Berlin, Germany \\ ${ }^{3}$ Laboratoire de Chimie Génétique associé au CNRS, \\ Université Louis Pasteur, Faculté de Pharmacie de \\ Strasbourg, F-67401 Illkirch, France \\ 4 Department of Physiology, \\ ${ }^{5}$ Department of Pediatrics, Ludwig-Maximilians- \\ University, D-80337 Munich, Germany \\ ${ }^{*}$ Corresponding author
}

In order to enhance and target gene delivery we have previously established a novel method, termed magnetofection, which uses magnetic force acting on gene vectors that are associated with magnetic particles. Here we review the benefits, the mechanism and the potential of the method with regard to overcoming physical limitations to gene delivery. Magnetic particle chemistry and physics are discussed, followed by a detailed presentation of vector formulation and optimization work. While magnetofection does not necessarily improve the overall performance of any given standard gene transfer method in vitro, its major potential lies in the extraordinarily rapid and efficient transfection at low vector doses and the possibility of remotely controlled vector targeting in vivo.

Key words: Gene Delivery/Gene Vectors/Magnetic Drug Targeting/Magnetic Nanoparticles/

Magnetofection.

\section{Introduction}

Nucleic acid delivery to cells in culture is to a large extent a diffusion-limited process if the nucleic acid shuttle (the vector) is of nanometric size, which is the case for viruses (Chuck et al., 1996) and many synthetic systems. Hence, apart from the major biological barriers including the plasma, endo/lysosomal and nuclear membranes, the limited contact of only a fraction of the applied vector dose with the target cells represents a major limitation to successful and efficient nucleic acid delivery. As a consequence, physical methods that accelerate vector particles toward the target cells have been successful in enhancing nucleic acid delivery. These methods include exploiting gravitational (Luo and Saltzman, 2000) or centrifugal force (Bunnell et al., 1995) which sediment particles on the cells and which are proportional to particle mass. Also convective flow of the transfection medium toward the target cell is a useful tool to enhance gene delivery (Chuck et al., 1996). Furthermore,these methods include biolistic methods (the 'gene gun' approach; Klein et al., 1992) and the application of electric fields ('electroporation', for a review see Somiari et al., 2000). Not surprisingly, one of the earliest nonviral gene delivery methods exploited gravity by the formation of precipitates from DNA and calcium phosphate (Chen and Okayama, 1988). Also with modern synthetic gene transfer systems, large and/or heavy vector particles were more efficient in gene delivery than small particles (Ogris et al., 1998; Luo and Saltzman, 2000). In addition to the mentioned biophysical barriers, also insufficient targetability of nucleic acid vectors to target cells/organs represents a limitation to their use in therapy or as research reagents. In the one extreme, limited targetability is due to the lack of a specific target cell tropism and as a consequence systemic spread of the vector if applied in vivo, limited bioavailability at a desired target site and the possibility of non-specific transfection of non-target cells/organs. In the other extreme, a very specific host tropism can limit the applicability of a vector to a broader target spectrum. This problem prevails for some viral vectors, both in ex- and in vivo gene delivery (Kim et al., 2002). A further limitation can be the rapid inactivation of vectors due to undesired interactions with components of the in vivo milieu before the vector can find its target site. Examples of undesired interactions are opsonization of nonviral vectors (Ogris et al., 1999) or interactions of vectors with host defense systems such as the complement (Plank et al., 1996) or the immune system (Kass-Eisler et al., 1996). Hence, there is an urgent need for simple, affordable and efficient tools for rapid accumulation of gene vectors at their target sites. It would be most desirable if vector accumulation could be remotely controlled by physical means, particularly in the case of intravenous or other systemic administration in vivo.

We have recently developed a method of magnetically 


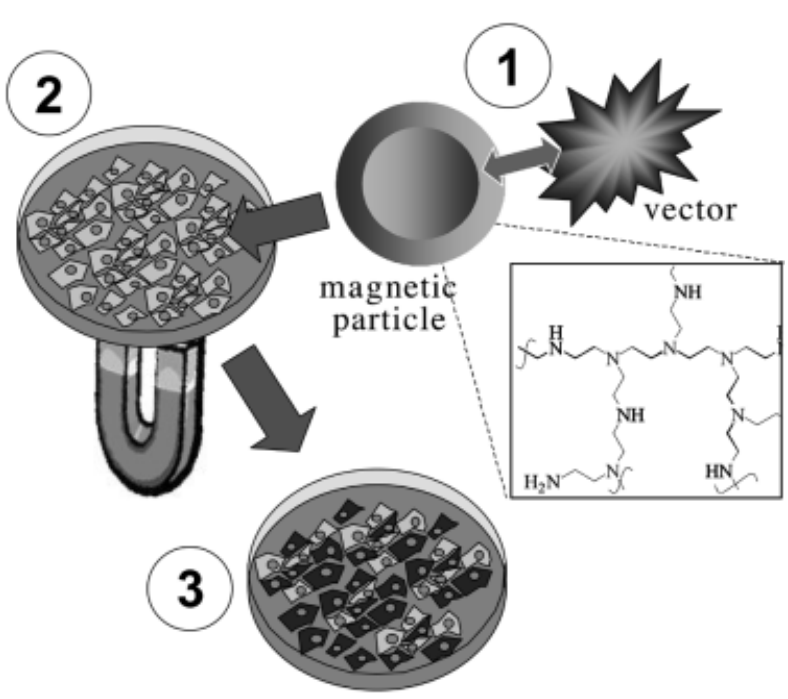

Fig. 1 Principle of Magnetofection.

Vectors and magnetic particles are associated by an appropriate linkage, in our case by salt-induced colloid aggregation (see Figure 2). For this purpose, magnetic particles were coated with polyelectrolytes, i.e. the polyelectrolyte is tightly bound to the particle surface. In most of the experiments shown here, polyethylenimine (PEI) was used as coating polyelectrolyte. If such particles are mixed with naked DNA, lipoplexes or polyplexes (such as PEI-DNA) in salt-containing buffer they will bind to or co-aggregate with these compounds. Cells are incubated with the vector-magnetic particle cocktail under the influence of a magnetic field which attracts the particles toward the cells. We have constructed magnetic plates from Nd-Fe-B permanent magnets (Scherer et al., 2002). The result of magnetofection is that essentially all cells get in contact with vectors and a high percentage of cells are rapidly transfected.

targeted nucleic acid delivery which we call magnetofection (Scherer et al., 2002): vectors are associated with superparamagnetic nanoparticles and accumulated on the target cells by the application of magnetic gradient fields (Figure 1, principle of magnetofection). We have shown that this approach is equally applicable to viral as well as nonviral gene vectors and that it potentiates and targets gene delivery in vitro as well as in vivo (Scherer et al., 2002). Our approach is based on previous work with low molecular weight drugs, mostly cytostatics, which, associated with magnetic particles, were accumulated in tumors and other tissues by the application of appropriate magnetic fields (Gupta and Hung, 1993; Lübbe et al., 1996; Lübbe and Bergemann, 1997, 1998; Alexiou et al., 2000). Other approved medical applications of magnetic particles include their use as contrast agents in magnetic resonance imaging, so that the pharmacokinetics and toxicity profiles of certain classes of iron oxide particles are well described (Weissleder et al., 1989). Pioneering work by Weissleder and coworkers has produced multiple applications of the technique, among these also imaging of transgene expression (Lewin et al., 2000; Weissleder et al., 2000; Wunderbaldinger et al., 2000).

\section{Magnetic Particle Chemistry and Physics}

The particles used in such approaches were mostly iron oxides, the synthesis of which is described mainly in the patent literature (see for example Pilgrimm, 1999) but also in text books (Schwertmann and Cornell, 1991). Briefly, such particles are derived by precipitation from acidic iron(II)/iron(III)-salt solutions upon addition of bases. Notably, also particles consisting of double oxides/hydroxides of two- or three valent iron with metal ions from the first row of transition metals, such as $\mathrm{Co}(\mathrm{II})$, $\mathrm{Mn}(\mathrm{II}), \mathrm{Cu}(\mathrm{II}), \mathrm{Ni}(\mathrm{II})$ or $\mathrm{Cr}$ (III), Gd(III), Dy(III), Sm(III), and furthermore finely milled metallic materials display the characteristics that make them suitable as magnetic carriers. To stabilize such particles they are coated with polymers, for use in biology preferably with biocompatible polymers of synthetic or natural origin. It is important to note that chemists were not the first to design such particles. Magnetite crystals are found in living organisms such as magnetotactic bacteria, in the central nervous systems of fish and even in the human brain (Kirschvink et al., 1992; Mertl, 1999; Diebel et al., 2000; Lohmann and Johnsen, 2000). At least for fish, magnetite has been defined as a magnetoreceptor essential for orientation along the earth's magnetic field (Diebel et al., 2000), and there is experimental evidence that similar mechanisms apply in migratory birds (Beason et al., 1995).

The term 'magnetic particle' used in the literature and also here generally refers to magnetically responsive solid phases, which are particles or aggregates thereof with micro- to nanometer size. The often used, but physically unprecise term 'magnetic', refers to temporarily magnetic materials, which can be ferrimagnetic or ferromagnetic. The term, however, also comprises paramagnetic and superparamagnetic materials. For the precise meaning of these terms the reader is referred to Fahlvik et al. (1993) or to text books of physics. The important characteristics for the application described here are that magnetic particles would orient in an externally applied magnetic field, an orientation (magnetization) that however would be immediately overcome by thermal motion upon removal of the external field. In order to have the particles move in a preferred direction of space, they must 'sense' an inhomogenous magnetic field ('gradient field'). The magnetic force acting on such particles in a liquid suspension is proportional to the particle volume, to the magnetic flux density and the magnetic field gradient (Zborowski et al., 1995). This relationship sets the framework that any usage of such particles has to cope with.

\section{Magnetofection - Magnetic Particles in Gene Delivery}

The conclusion that what is feasible with low molecular weight compounds should work for nucleic acids as well may appear trivial, however the implementation of such an approach requires a careful consideration of critical 
parameters: nucleic acids are charged macromolecules (polyelectrolytes) with their own very peculiar biophysical characteristics (Bloomfield, 1996). Unlike many low molecular weight drugs, they cannot cross the cellular membranes by simple diffusion, and if genes are to be delivered in the form of DNA, the molecules have to reach the nuclei of target cells. Hence, natural transport processes such as endocytosis have to be exploited to deliver these molecules, which holds also true if viruses are used as the nucleic acid shuttle. This means that the association of the vectors with magnetic particles must not interfere with the transport processes used. At the same time, the association has to be stable enough to endure the delivery process itself which in vivo involves interactions with host defense mechanisms and a multitude of solutes in blood and other body fluids. All this imposes certain requirements on the physical nature (size and surface characteristics) of the transport system as a whole, but also of its components, and also on the linkages that hold the components together. Provided that magnetic particles with appropriate reactive surface groups are available, physical, chemical and biological linkages can be envisaged. Among the latter, biotin-streptavidin and antigenantibody bridges have been successfully used to link viruses to nonviral vector particles (Curiel et al., 1991; Wagner et al., 1992) and more recently also to link viruses to magnetic particles (Hughes et al., 2001; Mah et al.,
2002; Pandori et al., 2002). The term nonviral vector particle refers to complexes of DNA with polycations ('polyplexes') or cationic lipids ('lipoplexes'). These complexes have been shown by numerous researchers to be particles of nanometric size or aggregates thereof. Often, toroids and rods are observed for polyplexes and less regular structures for lipoplexes, sometimes extending into the micrometer size range (an example of such structures is shown in Finsinger et al., 2000). As (strept)avidin or antibody-coated magnetic particles are expensive to manufacture, we decided to use the simplest way of linkage, physical interaction, which not only is the cheapest way but also highly effective and fully compatible with the cellular processing of gene vectors. Therefore we used magnetic particles coated with polyelectrolytes (in the following often referred to as 'magnetic particles') which are, in the same manner as many nonviral vector particles, subject to salt-induced aggregation (Figure 2A), a phenomenon well known in colloid science (Hiemenz, 1986). In this manner, simple mixing of polyelectrolytecoated magnetic particles and gene vectors at an appropriate ratio in salt-containing medium was sufficient to achieve quantitative association (Scherer et al., 2002). It is important to note that when pre-assembled nonviral vectors are associated with polyelectrolyte-coated magnetic particles, the vector and the magnetic particles do not need to have opposite surface charges. In fact, in
A

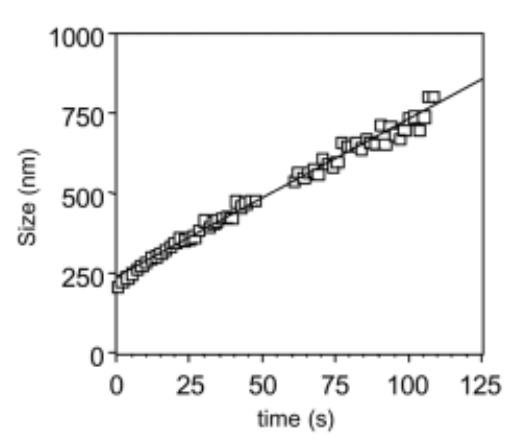

B

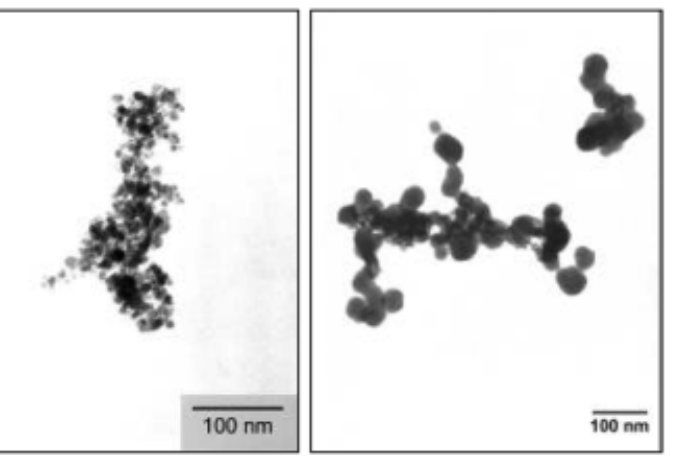

$\mathrm{C}$

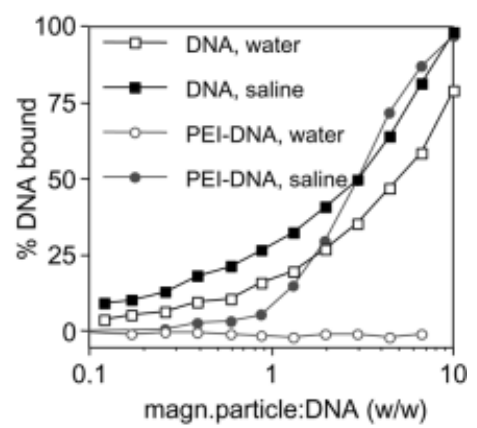

Fig. 2 Vector Association with Magnetic Particles: Salt-Induced Colloid Aggregation.

(A) A ternary complex was prepared in water by mixing DNA with PEl-coated magnetic particles and additional free PEl $(10 \mu \mathrm{gNA} / \mathrm{ml}$ final concentration; $25 \mathrm{kDa}$ PEl from Aldrich). Salt-induced aggregation was initialized by adjusting the ionic strenght to $150 \mathrm{~mm}$ sodium chloride. The complex aggregated with approx. linear kinetics starting at $217.0 \pm 2.0 \mathrm{~nm}$ and remaining in the sub-micrometer range within two hours (size measurement by dynamic light scattering using a Malvern $3000 \mathrm{HS}$ Zetasizer). (B) Electron microgaphs of PEIcoated magnetic particles (transMAG-PEI, left) and their associates with PEI-DNA vectors produced by salt-induced aggregation (right) as described in (A). The micrographs show that the magnetic particles are multi-domain structures of irregular shape. The resulting DNA complexes (right) display a tight association of PEI-DNA structures (gray/black spherical structures) with magnetic particles. Electron microscopy was performed as described by Erbacher et al. (1999). (C) DNA binding isotherms. DNA was labeled with [125I] according to the Commerford method modified as described by Terebesi et al. (1998). DNA (squares) or PEI-DNA complexes (circles; N/P ratio=8) were mixed with magnetic particles in water at increasing particle:DNA weight ratios, final DNA concentration $10 \mu \mathrm{g} / \mathrm{ml}$. The N/P ratio is the ratio of the nitrogen atoms of PEI to the phosphate groups of DNA in the complex. In water (open squares and circles) magnetic particles did not associate with PEI-DNA complexes but bound naked DNA. Upon adjustment of the ionic strength to $150 \mathrm{~mm}$ sodium chloride, binding of naked DNA was enhanced up to 1.7-fold at a given weigth ratio. A sigmoidal binding curve was obtained for PElDNA. The binding curves demonstrate the importance of salt-induced aggregation for vector-particle association and show that positively charged magnetic particles can easily be associated with positively charged gene vectors. Interestingly, PEI-DNA vectors displayed a similar binding behaviour with negatively charged magnetic particles (data not shown). 
most of our experiments we associated positively charged vector particles with positively charged magnetic particles (Figure 2). The appropriate ratios of magnetic particle and vector (or naked DNA) were found by serial titrations of radiolabeled DNA with magnetic particles followed by magnetic sedimentation and determination of the DNA fraction remaining in the supernatants (Figure $2 \mathrm{~B}$ ). The binding data were then set in correlation to gene transfer efficiency data obtained with multi-dimensional titrations of vector dose/magnetic-particle-to-DNA ratios/vector type in various standard cell lines. From such titrations we found numerous examples where magnetofection was clearly superior to standard trans-

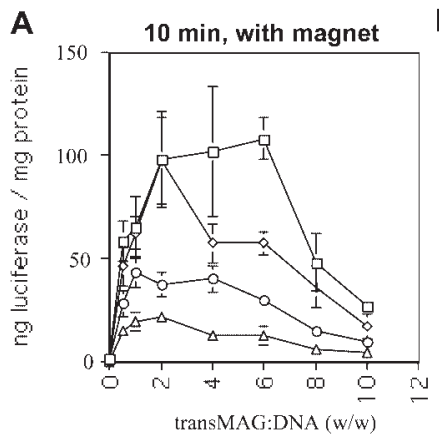

B $10 \mathrm{~min}$, without magnet
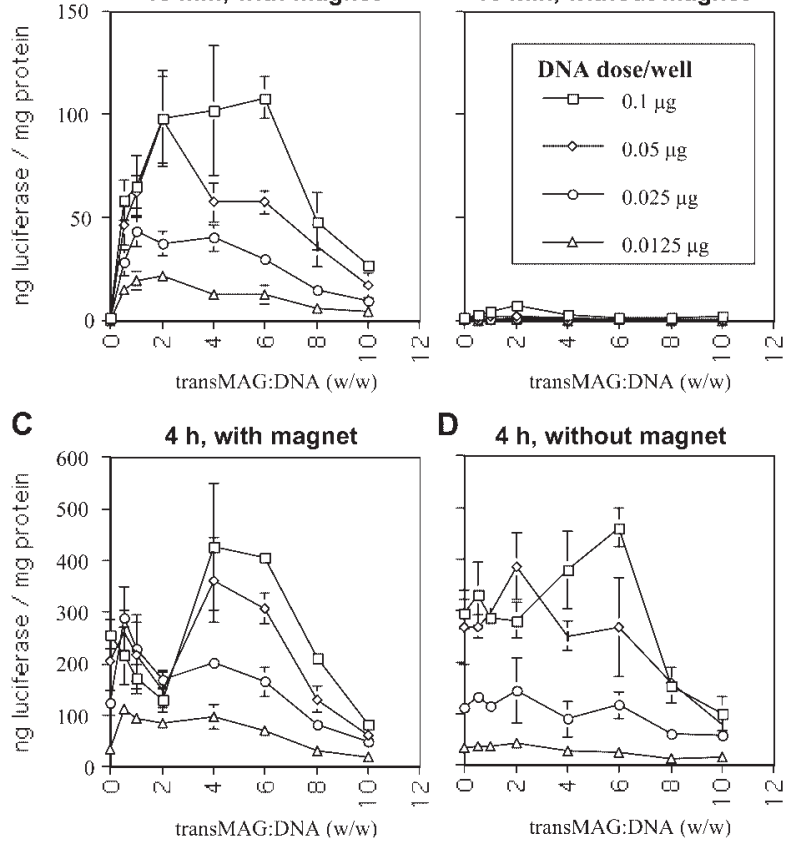

D

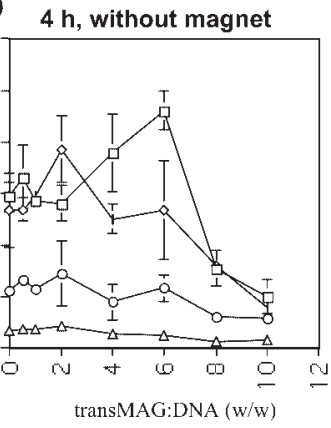

Fig. 3 Dose-Response Profiles of Magnetofection of CHO-K1 Cells with the GenePorter Reagent.

One vol. equivalents of DNA stock solutions in DMEM $(20 \mu \mathrm{g} / \mathrm{ml})$ were added to equal volumes each of a serial dilution of magnetic particles in DMEM, mixed, and then mixed immediately with two vol. equivalents GenePorter dilution $(50 \mu \mathrm{l} / \mathrm{ml}$ in DMEM). The samples were diluted with DMEM to a volume corresponding to 20 vol. equivalents, then a series of two-fold dilution steps in DMEM was performed in a 96-well plate. The medium from cells that were seeded in 4 plates at a density of 20000 cells/96 well was removed and $100 \mu$ leach of DNA complexes were added in triplicates. Two plates each were incubated as usual and two plates each on a magnetic plate for $10 \mathrm{~min}$ and 4 hours, respectively. The 10 min incubation plates were washed and then supplemented with fresh medium containing $10 \%$ FCS and penicillin/streptomycin. To the 4 hour plates $100 \mu \mathrm{l} /$ well of fresh medium containing $20 \%$ FCS (+ pen/strep) were added without a washing step. The luciferase assay was carried out as described (Finsinger et al., 2000) after 24 hours. The Figure shows that the transfection efficiency decreased at higher magnetic particle doses probably due to toxicity. A magnetic particle to DNA ratio of 4 turned out useful at all DNA doses. The data points represent the averages of triplicates \pm standard deviations. fection with the same vector (some examples are shown in Figures 6-9 and in Scherer et al., 2002). However, there were also examples where magnetofection was superior only in some but not all aspects. Figure 3 shows such an example with the lipofection reagent GenePorter (from Gene Therapy Systems, Inc., San Diego, USA) which is shown here because it highlights benefits and limitations of magnetofection in a single experiment. $\mathrm{CHO}-\mathrm{K} 1$ cells were transfected in the 96-well format with serial dilutions of vector compositions containing zero (standard vector) or increasing amounts of magnetic particles. The incubation times were either 10 minutes or 4 hours with or without exposure to a magnetic field, followed by washing of the cells. Figure $3 \mathrm{~A}$ versus $3 \mathrm{~B}$ shows the strong enhancement of transfection by the influence of the magnetic field during short time incubation and the failure of the standard vector to yield significant transfection levels during this short time. High magnetic particle content led to a drop in transfection efficiency. The drop was particularly pronounced at the higher DNA doses, probably due to toxicity. Comparing Figures $3 A$ and $B$ to $3 C$ and $D$ shows that magnetofection achieved the same order of magnitude of transgene expression level within 10 minutes of incubation as achievable within 4 hours (although an approximately 4-fold increase was obtained during 4 hours of incubation). Comparing Figure $3 \mathrm{C}$ and D shows first of all that long time exposure to the magnetic field did not yield major enhancements. In fact, depending on the exact vector composition and dose, the transfection without magnetic field was superior in some instances. Another important lesson from this experiment was that magnetofection of a cell line in vitro is not necessarily superior to the standard procedure in terms of the achievable overall transgene expression level. A close look at Figures $3 C$ and $D$ reveals that the standard transfection procedure was outdone by magnetofection only at the lower vector doses. Taken together, results shown in Figure 3 indicate that magnetofection is rapid in achieving very significant transfection levels, but that it requires fine tuning of vector choice, vector composition, vector dose and incubation time to exceed the overall expression levels obtained with some standard methods during long-time incubation.

It was an interesting finding that the mixing order of the vector components (DNA, polyelectrolyte-coated magnetic particles, third components such as cationic lipids or polyethylenimine) did not have a major influence on the observed transfection efficiencies. Neither was there a major difference in dependence on whether the surface coating of the magnetic particles was polycationic or polyanionic (an example is shown with the lipofection reagent DOTAP-cholesterol in Figure 4). Also, the transfection efficiency was largely independent of the type of polycation or polyanion used for particle coating (data not shown). Various coatings including PEI of various molecular weights, DEAE dextran, poly(aspartic acid), poly(maleic acid), poly(acrylic acid), or phosphate-derivatized starch were tested. This formulation work led us 


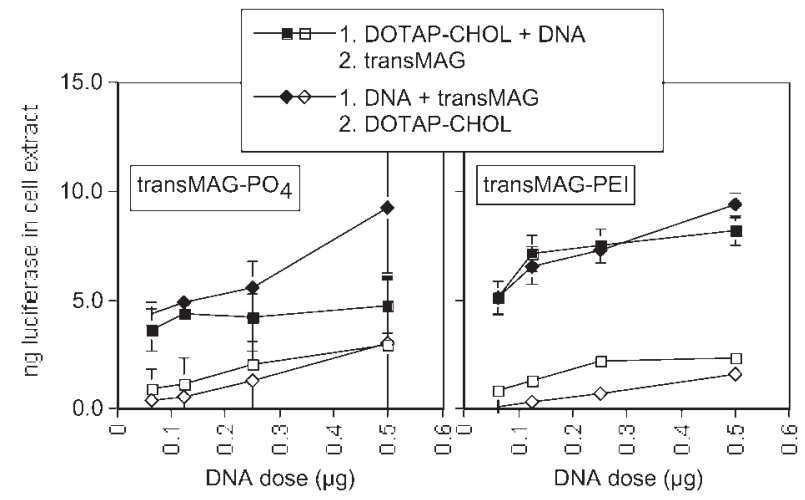

Fig. 4 Magnetofection of NIH 3T3 Cells Using the DOTAPCholesterol Reagent and Magnetic Particles with Positive and Negative Surface Charges.

DOTAP (1,2-dioleoyl-3-trimethylammoniumpropane) was purchased from Avanti Polar Lipids (Alabaster, AL, USA). DOTAP/ cholesterol (molar ratio 1:0.9) liposomes were prepared essentially as described (Meyer et al., 1995) as $5 \mathrm{~mm}$ (DOTAP) suspension in $5 \%$ glucose. Two mixing protocols were used. ( $\square, \square)$ : one vol. equivalent DNA stock solution in $\mathrm{HBS}(30 \mu \mathrm{g} / \mathrm{ml})$ was mixed with one vol. equiv. DOTAP/chol liposome stock (455 $\mu \mathrm{M}$ DOTAP in HBS) followed by mixing with one vol. equiv. magnetic particle suspensions $(30 \mu \mathrm{g} / \mathrm{ml})$. $(\diamond, \diamond)$ : using the same reagents, first magnetic particles and DNA were mixed and then added to DOTAP-chol. Magnetic particles were transMAG-PO4 (coating with phosphorylated starch), transMAG-PEI (coating with PEI). A dilution series was prepared to yield the indicated DNA doses per well ( $50 \mu$ l aliquots of DNA complexes were added per well in triplicates). Cells were seeded 7 hours prior transfection at a density of 30000 cells/well in $150 \mu \mathrm{l} \mathrm{DMEM} / 10 \%$ FCS/pen,strep. Per setup, experiments were carried out with (full symbols) and without positioning on a magnetic plate (open symbols) during $10 \mathrm{~min}$ of incubation followed by medium change.

to a simple protocol of magnetic vector formulation and application: the gene vector is prepared as usual with commercially available reagents according to the instructions of the manufacturer. In an additional step, the preformed vector is then mixed with an amount of magnetic particles such that the weight/weight ratio of magnetic particles and DNA will be 1:1 and higher. After an incubation time of 15 to $30 \mathrm{~min}$, the magnetic vectors are added to the cells in standard culture plates which are positioned on a magnetic plate (Scherer et al., 2002) for 15 minutes. After this time, the medium can either be changed or left on the cells until further evaluation (such as luciferase assay etc.). Magnetic plates for transfection purposes are commercially available (chemicell, Berlin, Germany) but can also be home-made with strong permanent magnets (Scherer et al., 2002). Similar plates are available mostly for cell or nucleic acid separation purposes from a number of suppliers (including Dexter, Promega, Miltenyi, Dynal) and may be useful for magnetofection, depending on the geometry of the magnet positioning. The essential requirement for magnetofection is that the magnetic particle-associated vectors experience a magnetic gradient field strong enough to pull them toward the cells to be transfected. As the fields of individual magnets in close proximity will influence each other, it is difficult to achieve an even distribution of magnetically sedimented vectors on the cells. On the other hand, with an appropriate positioning of magnets, it is easy to obtain a selective transfection of only part of a cell population within one well (Pandori et al., 2002; Scherer et al., 2002). While this already demonstrates the principle possibility of selective magnetic field-directed targeting for in vivo gene delivery, this feature may also be a useful technical tool for evaluating gene or protein function having the untransfected cell control within the same well.

\section{Mechanism and Benefits of Magnetofection}

The major efficacy-enhancing mechanism of magnetofection appears to be the rapid sedimentation of the full vector dose on the target cells, such that up to $100 \%$ of these cells will have vector particles bound to their surfaces within a few minutes (see for example Figure 9). Achieving the same frequency of vector-target cell contact may take hours during conventional transfection. Nevertheless, an additional effect of the magnetic field can not be excluded. It has been shown that upon injection of magnetic particles into rat tail veins and positioning of a strong permanent magnet downstream of the injection site, magnetic particles are found outside the blood vessel, implying that the magnetic field had caused extravasation into the surrounding tissue (Gupta and Hung, 1993). Therefore, it can be speculated that the magnetic field might pull the magnetic particle-vector associates across the plasma membrane into the cells. Also, as of yet unknown changes in cellular physiology upon exposition to the magnetic field may play a role. However, our results indicate that the primary uptake mechanism proceeds via the endo/lysosomal pathway, as we have argued before (Scherer et al., 2002). With naked DNA bound by electrostatic interaction to polycation-coated magnetic particles we only achieve low effiency gene transfer. When to this associate PEI, cationic lipids, a membrane-destabilizing agent such as the peptide INF7 (Plank et al., 1994) or an inactivated adenovirus (Cotten et al., 1994) are added, gene delivery is greatly enhanced. All these agents have been inferred to mediate endo/lysosomal release upon endocytotic vector uptake and therefore to enhance the transfection efficiency. Another strong evidence of endosomal uptake comes from electron microscopic studies that we have carried out (Figure 5). We found magnetic particles associated with a majority of the cells within 15 min of magnetic field exposure. We found particles in cellular invaginations resembling coated pits (Figure 5A). After 24 hours we often found the particles in membrane-surrounded structures which may be endosomes or lysosomes (Figure $5 B$ ). When the DNA component was labeled with colloidal gold, we found co-localization of magnetic parti- 


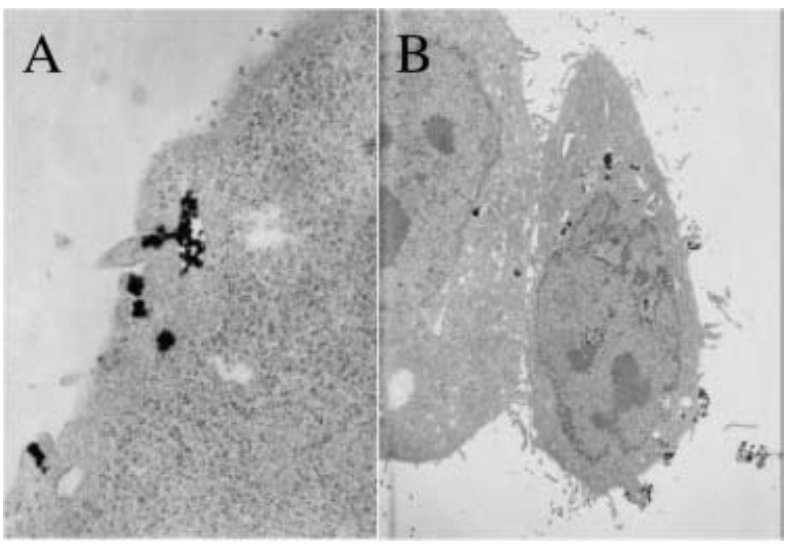

Fig. 5 Electron Micrographs of Magnetic Particle Complex Uptake in HeLa Cells.

HeLa cells were seeded in $35 \mathrm{~mm}$ dishes at a density of 300000 cells/well on the day prior transfection. Magnetic particle-DNA complexes were prepared at a particle:DNA ratio $(\mathrm{w} / \mathrm{w})$ of 2 $(4 \mu \mathrm{g} / \mathrm{ml}$ final DNA concentration in $5 \%$ glucose). The magnetic particle type used here were multi-domain iron oxide core coated with a multilayer of polyethylenimine ( $800 \mathrm{kDa}$, Fluka). Cells were kept in $1 \mathrm{ml}$ of medium, dishes placed on the magnetic plate, $250 \mu \mathrm{l}$ DNA complex were added and incubated for $15 \mathrm{~min}$. Cells were then washed gently and fixed in Sorensens buffer containing $1 \%$ glutaraldehyde for one hour, washed again, post-fixed in $1 \%$ aqueous osmium tetroxide, washed, and subjected to a dehydration series in graded ethanols. The samples were embedded in Epon. After sectioning, the samples were counter-stained with uranyl acetate and lead citrate. (A) Sample prepared directly after $15 \mathrm{~min}$ of magnetofection. (B) Sample prepared after 15 min magnetofection and 24 hours of further incubation. The pictures show endocytotic uptake of magnetic particle-DNA complexes. After 24 hours particles are frequently found inside cells, often in membrane-surrounded structures and even in the nucleus.

cles and DNA within the cells (data not shown). When we examined the influence of the duration of magnetic field exposure during magnetofection on the final transfection efficiency after 24 hours, we found that the mechanisms responsible for the observed high transfection efficiencies act during the first minutes of the magnetofection process. This is apparent from an experiment carried out with NIH 3T3 cells and the lipofection reagents Lipofectamine and GenePorter (Figure 6). For GenePorter-DNA associated with magnetic particles, exposing the cells to the vector under magnetic field influence only for five minutes was sufficient to yield $42 \%$ of the transfection level observed upon 4 hours of incubation, after $40 \mathrm{~min}$ already $73 \%$ of this level were reached, while 5 min standard transfection with the magnetic particle-free parent vector yielded only $0.04 \%$ and 4 hours yielded $20 \%$, respectively, of the reporter gene expression level achieved with magnetofection during 4 hours. For GenePorter, the kinetics of the magnetofection process displayed a logarithmic profile, while the process with the same vector in the absence of a magnetic field or with the standard vector displayed a linear relationship between luciferase ex- pression and incubation time of cells with the vector cocktail. The same experiment carried out with Lipofectamine (Figure 6A and $\mathrm{C}$ ) resulted in even decreasing transfection efficiency with increasing incubation time in the case of magnetofection, while the standard vector displayed a minor linear increase but being rather inefficient overall. Taken together, the transfection kinetics is another indication that the major enhancing factor in magnetofection is the rapid accumulation of the full vector dose at the target cell surface. This also led to a very early onset of reporter gene expression (as shown in Figure 7 for PEI-DNA complexes). Within 2 hours after a 15 minute magnetofection period, significant reporter gene expression levels were detectable, while it took between 4 and 8 hours to reach the minimal detectable expression level by standard transfection. For some vectors, another beneficial consequence of the rapid cell binding of the full vector dose was the highly favorable dose-response profile of magnetofection compared to standard transfection. For example, logarithmic profiles were observed for magnetofection with PEI, DOTAPCholesterol or Lipofectamine in contrast to a linear profile for standard transfection (Figure 4, DOTAP-Cholesterol; Figure 6, Lipofectamine). This means that by applying magnetofection, high transgene expression levels can be achieved at a very low vector dose with extremely short incubation times. This allows avoiding toxicity with cells that are sensitive to the transfection process (Krötz et al., 2003). High transgene expression levels are either achieved by having a few cells expressing tremendous amounts of the transfected gene or by having a high percentage of target cells transfected. In most of our magnetofection experiments we observed a combination of both, a high percentage of transfected cells producing high amounts of transgene product (as shown in Figure 8 for CT26 colon carcinoma cells transfected with DMRIEC and as also shown in Scherer et al., 2002, for adenoviral and retroviral magnetofection). Despite this success it needs to be emphasized that the final transgene expression level achieved with magnetofection is not necessarily higher in all cases than the one achieved under optimized conditions with a given standard transfection protocol (as is also evident from Figures 3 and 6). Like with all gene delivery methods, this is dependent on the cells to be transfected and on the skills and the awareness of the experimentator. In summary, the benefits of magnetofection are that (i) it saves time, (ii) it saves material, (iii) it can potentiate the efficacy of a given vector, and (iv) it allows magnetic field-guided targeting.

\section{Applications of Magnetofection}

These features make magnetofection a very useful tool where any one or several of these key points limit the success of a given approach involving nucleic acid delivery. There are multiple examples of such technical challenges: from gene therapy, where nucleic acid delivery it- 
A

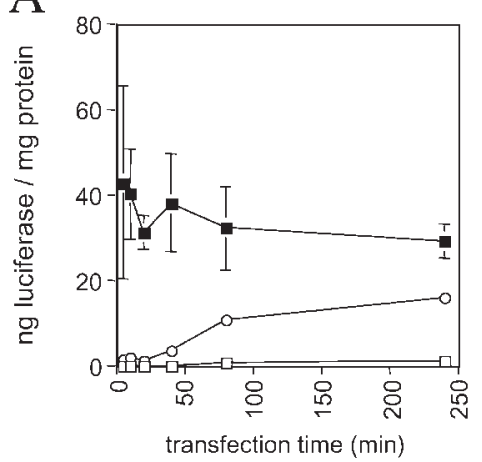

C

\section{$\longrightarrow$ magnetofection}

$\longrightarrow$ standard transfection

- - transMAG complex without magnetic field

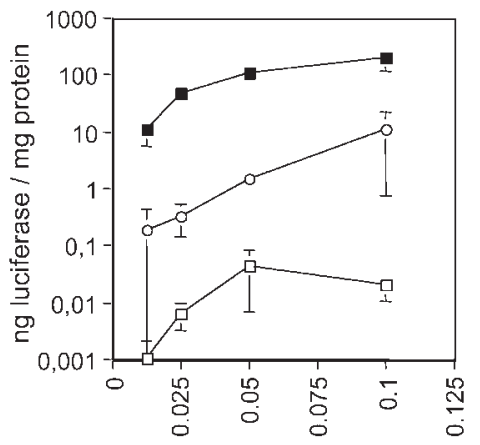

$\mu g$ DNA

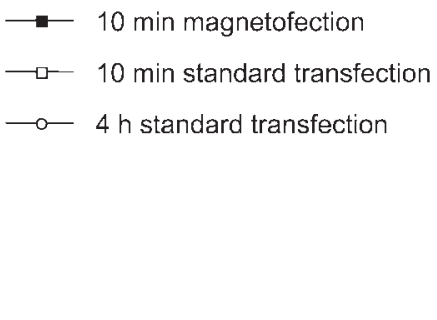

- 4 h standard transfection
B

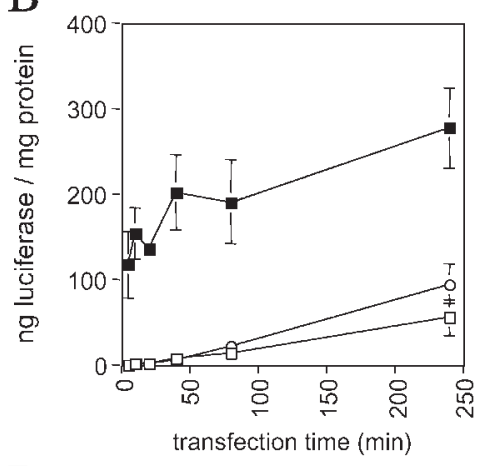

D

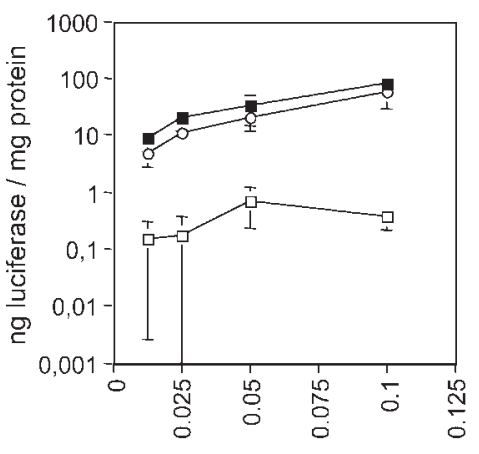

$\mu \mathrm{g}$ DNA

Fig. 6 Transfection Kinetics and Dose-Response Profile of Standard Transfection and Magnetofection in NIH 3T3 Cells Using the Reagents Lipofectamine and GenePorter.

The experiment was set up such as the one shown in Figure 3 with a fixed magnetic particle:DNA ratio of 2:1 (w/w), a DNA dose of $100 \mathrm{ng} /$ well for the kinetics study in (A) and (B), and a serial dilution for the dose-response study in (C) and (D), and with $4 \mu$ l of Lipofectamine and $5 \mu \mathrm{l}$ of GenePorter used per $\mu \mathrm{g}$ of DNA, respectively. The experiments shown in (A) and (B) were carried out with one culture plate each positioned upon the magnetic plate throughout the maximum incubation time of 240 minutes, one plate was kept under standard conditions. The vector formulations were removed from individual wells in triplicates each and cells in these wells were washed after 5, 10, 20, 40, and 240 minutes of incubation. The luciferase assay was carried out 20 hours after the start of the experiment. Under the conditions tested, maximum expression was found already after 5 min with Lipofectamine, while transfection efficiency increased over time but with a moderate slope with GenePorter ( $42 \%$ of the final reporter gene expression level was already achieved after $5 \mathrm{~min}$ ). Consistently, the Figures show that $10 \mathrm{~min}$ of magnetofection are sufficient to achieve the transfection levels obtained with the standard reagent using 4 hours of transfection. At any time point, GenePorter formulations were more efficient than Lipofectamine formulations.

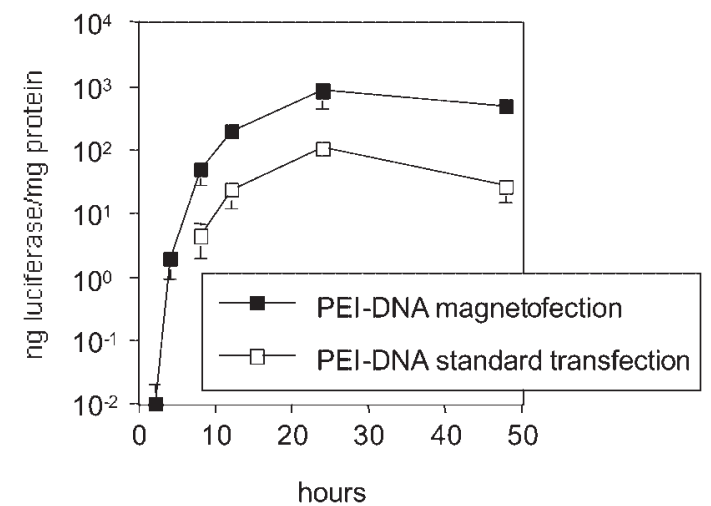

self is at the heart of the therapeutic strategy, to basic research or even commercial scale biotechnology approaches where nucleic acid delivery only serves as a tool for answering scientific questions. An example for the latter is a particular functional genomics approach where known or unknown DNA or RNA sequences are transfected into cells in a high throughput manner in order to obtain novel screenable phenotypes (see for ex-
Fig. 7 Reporter Gene Expression Kinetics of Magnetofected or Standard PEI-Transfected Luciferase Plasmid.

One vol. equivalent of a DNA stock solution $(60 \mu \mathrm{g} / \mathrm{ml})$ was mixed with one vol. equivalent of a PEl stock solution $(62.6 \mu \mathrm{g} / \mathrm{ml}, 25 \mathrm{kDa}$ branched PEI, Aldrich) or one vol. equivalent of a magnetic particle suspension $(240 \mu \mathrm{g} / \mathrm{ml})$, respectively (all dilutions in $0.9 \%$ sodium chloride). After 15 min of incubation one vol. equiv. of $0.9 \%$ sodium chloride was added to the PEI-DNA complexes and one vol. equiv. of PEl stock solution was added to the magnetic particleDNA complexes. After 15 min of incubation, $50 \mu$ leach (DNA dose $1 \mu \mathrm{g}$ ) of the resulting complexes were added to the cells seeded in two 96-well plates at a densitiy of 20000 cells/well, covered with $150 \mu$ fresh medium (DMEM, 10\% FCS). One plate was positioned on the magnetic plate throughout the first 8 hours of incubation, after which a medium change was performed (DMEM, 10\% FCS). During this incubation time, cells in triplicate wells each were washed with PBS and lysed for luciferase assay after 1, 2, 4, and 8 hours of incubation and then again after 24 and 48 hours. The luciferase assay was performed as described (Finsinger et al., 2000). The figure shows the early onset of detectable reporter gene expression upon magnetofection which peaked after 24 hours and which was consistently higher than the one achieved with standard transfection (enhancement of 9- to 18-fold after onset of reporter gene expression in the standard wells). 
ample Grimm et al., 2002). On the one hand this would allow to assign function to a given nucleic acid sequence, on the other hand it would allow to screen a given nucleic acid pool for therapeutically useful sequences, and in a further step to optimize such sequences in terms of a desired biological effect. Whatever the goal of such an approach may be, it would require thousands of successful transfections a day carried out in an automated manner. Process time, material consumption (and connected costs) and transfection efficiency represent the key bottle necks to such strategies. It can easily be envisaged to incorporate the magnetofection technology in a robotic system which would provide the solution to this technical challenge.

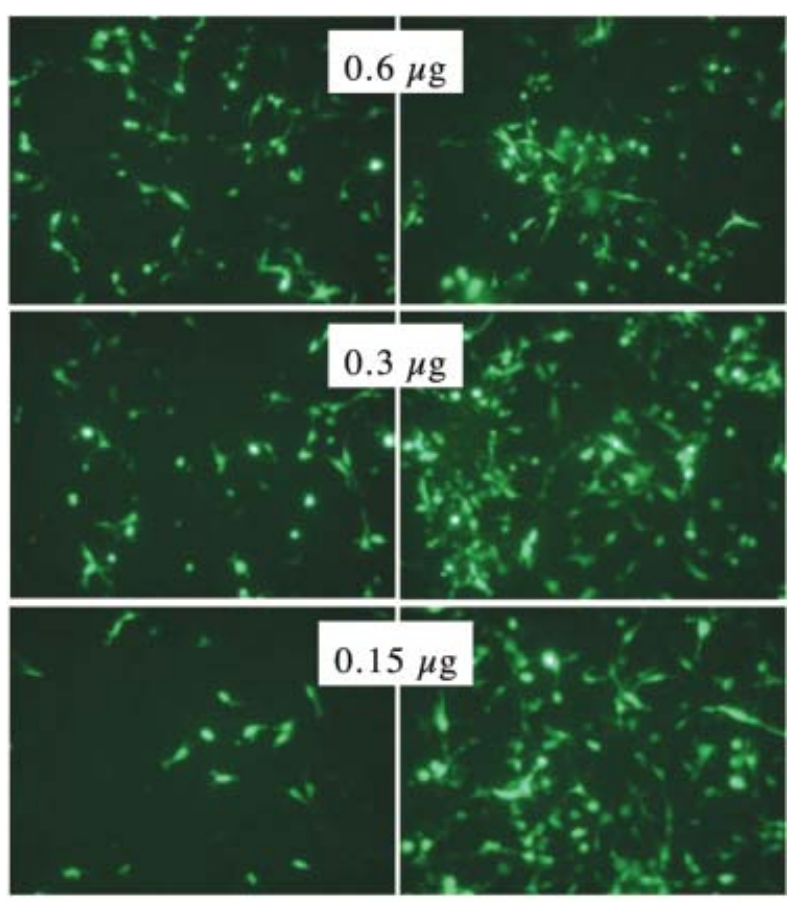

\section{standard transfection magnetofection}

Fig. 8 Magnetofection and Standard Transfection of the Colon Carcinoma Cell Line CT26 with the Transfection Reagent DMRIE-C Using Green Fluorescent Protein Gene as Reporter.

One vol. equiv. DNA stock ( $12 \mu \mathrm{g} / \mathrm{ml}$ in Optimem) was mixed with 2 vol. equiv. DMRIE-C suspension ( $30 \mu \mathrm{l} / \mathrm{ml}$ in Optimem), followed by mixing with one vol. equiv. magnetic particles $(12 \mu \mathrm{g} / \mathrm{ml}$ in Optimem) or with Optimem alone. A dilution series was prepared after 30 min of incubation. Cells (seeded in 24 well plates, supplemented with $200 \mu \mathrm{l} /$ well fresh Optimem prior transfection) were transfected by addition of $200 \mu$ l of complexes, containing DNA doses of 600,300 and $150 \mathrm{ng}$ DNA. The culture plate was positioned on a magnetic plate during the first $15 \mathrm{~min}$ of incubation, which was continued for a total of 5 hours. Then the medium was exchanged for RPMI1640 medium containing 10\% FCS. Fluorescence images were taken 24 hours after complex addition. The Figure shows the strong improvement in percentage of transfected cells which is particularly apparent at the lower DNA doses. Magnetofection of $150 \mathrm{ng}$ DNA resulted in higher efficiency than with $600 \mathrm{ng}$ DNA in standard vector formulation.

Our own long-term goal is the use of magnetofection as a tool for gene therapy, on the one hand for ex vivo approaches potentially useful in tissue engineering or for the generation of tumor vaccines, on the other hand for localized gene delivery in vivo with potential applications in cardio-vascular or, again, cancer therapy. Any of these purposes would involve the transfection of primary cells with the major technical challenges of efficacy and of targeting. Consequently, we have recently focussed our attention on establishing the technique in primary cells with reporter genes. We have shown that the association of adenovirus with magnetic particles provides the virus with the ability to infect cells which are non-permissive to adenoviral gene delivery because they lack the required virus receptor (CAR) (Scherer et al., 2002). This is important because the absence of this receptor in natural targets of adenoviral gene therapy, such as tumors, severely limits the feasibility of conceptionally highly promising approaches (Kim et al., 2002). Similarly, we have shown localized adenoviral and nonviral gene delivery by magnetofection in vivo in blood vessels and in the gastro-intestinal tract (Scherer et al., 2002). Our most recent results show that nonviral magnetofection is highly efficient in the ex vivo transfection of primary cells including hu-

A

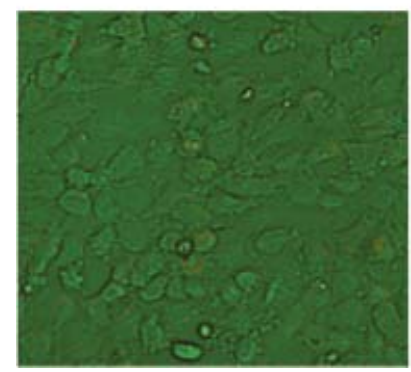

Fig. 9 Magnetofection and Standard Transfection of Antisense Oligonucleotides in HUVECs.

Cy3-labeled anti-SHP-1 phosphorothioate oligodeoxynucleotides (ODN) were transfected using Effectene ${ }^{\mathrm{TM}}$ in combination with magnetofection. Antisense-ODN (200 ng per well of a 24well plate, ca. $60 \mathrm{~nm}$ ) were mixed with buffer EC (volume added to result in a final volume of $30 \mu \mathrm{l}$ after step (1), Effectene $\mathrm{e}^{\mathrm{TM}}$ enhancer solution ( $\mathrm{w} / \mathrm{v}$ ratio of 8$)$ and magnetic particles (B) $(1 \mathrm{mg} / \mathrm{ml}$ suspension; w/w ratio of 1 ) or water only (A), vortexed for 1 second, and incubated for 2-5 minutes (step 1). Then, Effectene ${ }^{\mathrm{TM}}(\mathrm{w} / \mathrm{v}$ ratio of 20) was added, followed by vortexing for 10 seconds and incubation for $5-10$ minutes, and terminated by addition of medium 199 supplemented with $20 \%$ NBCS and antibiotics (HUVEC medium) to a final volume of $200 \mu \mathrm{l}$ (transfection mixture). Before addition of the transfection mixture (200 $\mu$ l each) to confluent HUVEC, the cells were washed once with phosphate buffered saline (PBS) and supplemented with $200 \mu \mathrm{l}$ of HUVEC medium. The culture plates were positioned on magnetic plates for 15 minutes, followed by washing the cells 3 times with PBS and continued cultivation in medium for 2 hours. Images were taken using an inverted laser scanning microscope (LSM 410, Zeiss, Jena, Germany). (A) Standard transfection; (B) magneto- 
man umbilical vein endothelial cells (HUVEC), porcine aortic endothelial cells (PAEC) (Krötz et al., unpublished results), rat carotid artery smooth muscle cells, explanted porcine veins, porcine and rabbit articular chondrocytes, human and porcine airway epithelial cells, intact porcine tracheal airway epithelium, and human keratinocytes (unpublished results). We were also successful in applying magnetic particle-DNA complexes as aerosol directly on human airway epithelial cells in culture with subsequent efficient transfection by magnetic field influence, giving a novel perspective to gene delivery to the lungs via the airways (J. Rosenecker, unpublished results).

We have used magnetofection with great success not only for the delivery of viruses and plasmid DNA but also for the delivery of antisense oligonucleotides (Krötz et al., 2003). The goal of this study was to define the role of the p22 ${ }^{\text {phox }}$ subunit of endothelial $\mathrm{NAD}(\mathrm{P}) \mathrm{H}$-oxidase in superoxide production in primary endothelial cells by an antisense knock-out approach. Measuring such a sensitive cellular function required that the oligonucleotide was delivered at low dose, rapidly, and that a high percentage of the target cells (HUVEC) were transfected. We found that by magnetofection, oligonucleotides, applied at low dose, became associated within minutes with up to $100 \%$ of the target cells (Figure 9), and were then rapidly internalized and accumulated in the nuclei. This resulted in the efficient knock-out of the target which then allowed us to demonstrate the important role of the p22 ${ }^{\text {phox }}$ subunit of endothelial $\mathrm{NAD}(\mathrm{P}) \mathrm{H}$-oxidase in the enzyme's basal activity. Conventional transfection was associated with significant toxicity as it either required a high vector dose and/or long incubation times (24 hours) to yield the required efficiency. However, when measuring sensitive physiological functions such as superoxide production, the scientific question itself is incompatible with transfection-associated toxicity as it would have a direct impact on the cellular function to be measured. In the same study (Krötz et al., 2003) we demonstrated the general applicability of antisense magnetofection with two additional independent targets of antisense knock-out (connexin 37 and cytosolic phosphatase SHP-1; data not shown here). Finally, we also demonstrated the feasibility of magnetic field-guided oligonucleotide targeting to blood vessels in vivo which in the future will allow the examination of gene function directly in vivo with having the untreated control blood vessels in the same animal (data not shown).

\section{Conclusions and Outlook}

During the last fifteen years we have witnessed the development of highly efficient tools for gene delivery to eukaryotic cells and more recently also their successful use in gene therapy (Aiuti et al., 2002a,b; Fischer et al., 2002). Despite these impressive achievements it may take additional decades until gene therapy becomes a broadly applicable therapeutic modality, particularly considering the many unknown factors and risks associated with any novel form of treatment. But this apparent delay with respect to original expectations is also due to unmet technical requirements, among other things relating to gene vector efficacy, safety and economic feasibility of its production and handling. Although the technique of magnetofection is far from providing a single general solution to these deficiencies, it opens a novel perspective on this stony path. First of all, it provides a technical solution for the rapid and efficient introduction of nucleic acids into primary cells which will greatly facilitate and accelerate the examination of gene function, the identification of nucleic acids with therapeutic potential and also the assessment of risks associated with the transfer of genetic material into cells. Secondly, the technique can become an option for in vivo targeting combined with improved efficacy of gene delivery, potentially in combination with other physical techniques, if appropriate magnetic fields can be generated. As mentioned before, the essential physical parameters governing the force acting on magnetic particles in liquid suspension are the magnetic flux density, the magnetic field gradient and the particle volume. Optimizing these parameters for biomedical applications raises several demanding engineering challenges in this exciting field of interdisciplinary research.

\section{References}

Aiuti, A., Slavin, S., Aker, M., Ficara, F., Deola, S., Mortellaro, A., Morecki, S., Andolfi, G., Tabucchi, A., Carlucci, F. et al. (2002a). Correction of ADA-SCID by stem cell gene therapy combined with nonmyeloablative conditioning. Science 296 , $2410-2413$.

Aiuti, A., Vai, S., Mortellaro, A., Casorati, G., Ficara, F., Andolfi, G., Ferrari, G., Tabucchi, A., Carlucci, F., Ochs, H. D., Notarangelo, L. D. et al. (2002b). Immune reconstitution in ADASCID after PBL gene therapy and discontinuation of enzyme replacement. Nature Med. 8, 423-425.

Alexiou, C., Arnold, W., Klein, R. J., Parak, F. G., Hulin, P., Bergemann, C., Erhardt, W., Wagenpfeil, S. and Lubbe, A. S. (2000). Locoregional cancer treatment with magnetic drug targeting. Cancer Res. 60, 6641-6648.

Beason, R., Dussourd, N. and Deutschlander, M. (1995). Behavioural evidence for the use of magnetic material in magnetoreception by a migratory bird. J. Exp. Biol. 198, 141-146.

Bloomfield, V. A. (1996). DNA Condensation. Curr. Opin. Struct. Biol. 6, 334-341.

Bunnell, B. A., Muul, L. M., Donahue, R. E., Blaese, R. M. and Morgan, R. A. (1995). High-efficiency retroviral-mediated gene transfer into human and nonhuman primate peripheral blood lymphocytes. Proc. Natl. Acad. Sci. USA 92, 7739-7743.

Chen, C. A. and Okayama, H. (1988). Calcium phosphate-mediated gene transfer: a highly efficient transfection system for stably transforming cells with plasmid DNA. Biotechniques 6 , $632-638$.

Chuck, A. S., Clarke, M. F. and Palsson, B. O. (1996). Retroviral infection is limited by Brownian motion. Hum. Gene Ther. 7, $1527-1534$.

Cotten, M., Saltik, M., Kursa, M., Wagner, E., Maass, G. and Birnstiel, M. L. (1994). Psoralen treatment of adenovirus particles eliminates virus replication and transcription while main- 
taining the endosomolytic activity of the virus capsid. Virology 205, 254-261.

Curiel, D. T., Agarwal, S., Wagner, E. and Cotten, M. (1991). Adenovirus enhancement of transferrin polylysine-mediated gene delivery. Proc. Natl. Acad. Sci. USA 88, 8850-8854.

Diebel, C. E., Proksch, R., Green, C. R., Neilson, P. and Walker, M. M. (2000). Magnetite defines a vertebrate magnetoreceptor. Nature 406, 299-302.

Erbacher, P., Remy, J.-S. and Behr, J.-P. (1999). Gene transfer with synthetic virus-like particles via the integrin-mediated endocytosis pathway. Gene Ther. 6, 138-145.

Fahlvik, A. K., Klaveness, J. and Stark, D. D. (1993). Iron oxides as MR imaging contrast agents. J. Magn. Reson. Imaging 3, $187-194$.

Fischer, A., Hacein-Bey, S. and Cavazzana-Calvo, M. (2002). Gene therapy of severe combined immunodeficiencies. Nature Rev. Immunol. 2, 615-621.

Finsinger, D., Remy, J. S., Erbacher, P., Koch, C. and Plank, C. (2000). Protective copolymers for nonviral gene vectors: synthesis, vector characterization and application in gene delivery. Gene Ther. 7, 1183-1192.

Grimm, S. and Kachel, V. (2002). Robotic high-throughput assay for isolating apoptosis-inducing genes. Biotechniques 32, 670-672, 674-677.

Gupta, P. K. and Hung, C. T. (1993). Magnetically controlled targeted chemotherapy. In: Microspheres and Regional Cancer Therapy, N. Willmott and J. Daly, eds. (Boca Raton, USA: CRC Press), pp. 71-116.

Hiemenz, P. C. (1986). Principles of Colloid and Surface Chemistry (New York, USA: Marcel Dekker, Inc.).

Hughes, C., Galea-Lauri, J., Farzaneh, F. and Darling, D. (2001). Streptavidin paramagnetic particles provide a choice of three affinity-based capture and magnetic concentration strategies for retroviral vectors. Mol. Ther. 3, 623-630.

Kass-Eisler, A., Leinwand, L., Gall, J., Bloom, B. and Falck-Pedersen, E. (1996). Circumventing the immune response to adenovirus-mediated gene delivery. Gene Ther. 3, 154-162.

Kim, M., Zinn, K., Barnett, B., Sumerel, L., Krasnykh, V., Curiel, D. and Douglas, J. (2002). The therapeutic efficacy of adenoviral vectors for cancer gene therapy is limited by a low level of primary adenovirus receptors on tumour cells. Eur. J. Cancer 38, 1917.

Kirschvink, J. L., Kobayashi-Kirschvink, A. and Woodford, B. J. (1992). Magnetite biomineralization in the human brain. Proc. Natl. Acad. Sci. USA 89, 7683-7687.

Klein, T. M., Arentzen, R., Lewis, P. A. and Fitzpatrick-McElligott, S. (1992). Transformation of microbes, plants and animals by particle bombardment. Biotechnology 10, 286-291.

Krötz, F., de Wit, C., Sohn, H.-Y., Zahler, S., Gloe, T., Pohl, U. and Plank, C. (2003). Definition of an important role of p22phox in endothelial superoxide production by magnetofection - a highly efficient tool for oligonucleotide delivery in vitro and in vivo. Mol. Ther., in press.

Lewin, M., Carlesso, N., Tung, C. H., Tang, X. W., Cory, D., Scadden, D. T. and Weissleder, R. (2000). Tat peptide-derivatized magnetic nanoparticles allow in vivo tracking and recovery of progenitor cells. Nature Biotechnol. 18, 410-414.

Lohmann, K. J. and Johnsen, S. (2000). The neurobiology of magnetoreception in vertebrate animals. Trends Neurosci. 23, $153-159$.

Lübbe, A. S., Bergemann, C., Riess, H., Schriever, F., Reichardt, P., Possinger, K., Matthias, M., Dorken, B., Herrmann, F., Gurtler, R. et al. (1996). Clinical experiences with magnetic drug targeting: a phase I study with 4'-epidoxorubicin in 14 patients with advanced solid tumors. Cancer Res. 56, 4686-4693.

Lübbe, A. S. and Bergemann, C. (1997). Selected preclinical and first clinical experiences with magnetically targeted 4-epidoxorubicin in patients with advanced solid tumors. In: Scientific and Clinical Applications of Magnetic Drug Carriers, U. Häfeli, W. Schütt, J. Teller and M. Zborowski, eds. (New York, London: Plenum Press).

Lübbe, A. S. and Bergemann, C. (1998). Magnetically controlled drug targeting. Cancer J. 11, 104-105.

Luo, D. and Saltzman, W. M. (2000). Enhancement of transfection by physical concentration of DNA at the cell surface. Nature Biotechnol. 18, 893-895.

Mah, C., Fraites, T. J. J., Zolotukhin, I., Song, S., Flotte, T. R., Jon Dobson, Batich, C. and Byrne, B. J. (2002). Improved method of recombinant AAV2 delivery for systemic targeted gene therapy. Mol. Ther. 6, 106-112.

Mertl, M. (1999). Magnetic cells: stuff of legend? Science 283, 775.

Meyer, K. B., Thompson, M. M., Levy, M. Y., Barron, L. G. and Szoka, F. C., Jr. (1995). Intratracheal gene delivery to the mouse airway: characterization of plasmid DNA expression and pharmacokinetics. Gene Ther. 2, 450-460.

Ogris, M., Steinlein, P., Kursa, M., Mechtler, K., Kircheis, R. and Wagner, E. (1998). The size of DNA/transferrin-PEI complexes is an important factor for gene expression in cultured cells. Gene Ther. 5, 1425-1433.

Ogris, M., Brunner, S., Schuller, S., Kircheis, R. and Wagner, E. (1999). PEGylated DNA/transferrin-PEI complexes: reduced interaction with blood components, extended circulation in blood and potential for systemic gene delivery. Gene Ther. 6 , $595-605$.

Pandori, M. W., Hobson, D. A. and Sano, T. (2002). Adenovirusmicrobead conjugates possess enhanced infectivity: a new strategy to localized gene delivery. Virology 299, 204-212.

Pilgrimm, H. (1999). Superparamagnetic particles, process for producing the same and their use. US Patent No. 5,916,539; Silica Gel GmbH.

Plank, C., Oberhauser, B., Mechtler, K., Koch, C. and Wagner, E. (1994). The influence of endosome-disruptive peptides on gene transfer using synthetic virus-like gene transfer systems. J. Biol. Chem. 269, 12918-12924.

Plank, C., Mechtler, K., Szoka, F. C. and Wagner, E. (1996). Activation of the complement system by synthetic DNA complexes: a potential barrier for intravenous gene delivery. Hum. Gene Ther. 7, 1437-1446.

Scherer, F., Anton, M., Schillinger, U., Henke, J., Bergemann, C., Kruger, A., Gansbacher, B. and Plank, C. (2002). Magnetofection: enhancing and targeting gene delivery by magnetic force in vitro and in vivo. Gene Ther. 9, 102-109.

Schwertmann, U. and Cornell, R. M. (1991). Iron Oxides in the Laboratory. Preparation and Characterization (Weinheim, Germany: Wiley/VCH).

Somiari, S., Glasspool-Malone, J., Drabick, J. J., Gilbert, R. A., Heller, R., Jaroszeski, M. J. and Malone, R. W. (2000). Theory and in vivo application of electroporative gene delivery. Mol. Ther. 2, 178-187.

Terebesi, J., Kwok, K. Y. and Rice, K. G. (1998). lodinated plasmid DNA as a tool for studying gene delivery. Anal. Biochem. 263, 120-123.

Wagner, E., Zatloukal, K., Cotten, M., Kirlappos, H., Mechtler, K., Curiel, D. T. and Birnstiel, M. L. (1992). Coupling of adenovirus to transferrin polylysine DNA complexes greatly enhances receptor-mediated gene delivery and expression of transfected genes. Proc. Natl. Acad. Sci. USA 89, 6099-6103.

Weissleder, R., Stark, D. D., Engelstad, B. L., Bacon, B. R., Compton, C. C., White, D. L., Jacobs, P. and Lewis, J. (1989). Superparamagnetic iron oxide: pharmacokinetics and toxicity. Am. J. Roentgenol. 152, 167-173. 
Weissleder, R., Moore, A., Mahmood, U., Bhorade, R., Benveniste, H., Chiocca, E. A. and Basilion, J. P. (2000). In vivo magnetic resonance imaging of transgene expression. Nature Med. 6, 351-355.

Wunderbaldinger, P., Bogdanov, A. and Weissleder, R. (2000).
New approaches for imaging in gene therapy. Eur. J. Radiol. 34, 156-165.

Zborowski, M., Fuh, C. B., Green, R., Sun, L. and Chalmers, J. J. (1995). Analytical magnetapheresis of ferritin-labeled lymphocytes. Anal. Chem. 67, 3702-3712. 